\title{
Asignaturas pendientes del DSM-5
}

\author{
Josep Artigas-Pallarés, Isabel Paula-Pérez
}

Resumen. El presente artículo analiza las críticas generadas a partir de la publicación del Manual diagnóstico y estadístico de los trastornos mentales, quinta edición (DSM-5), ya anunciadas parcialmente durante las últimas fases de su elaboración. Una parte de las críticas se ha centrado en los cambios de los criterios diagnósticos para determinados trastornos y en la incorporación al DSM de nuevas entidades. Sin embargo, otra vertiente crítica va dirigida a la falta de validez de los diagnósticos del DSM, por cuyo motivo se ha cuestionado su eficiencia en el campo de la investigación. El fallo básico del DSM se centra en la incoherencia de un modelo basado en un amplio repertorio de definiciones de entidades categóricas, todas ellas con un alto componente de comorbilidad. Como propuesta para superar el bloqueo generado en la investigación y la parquedad de avances terapéuticos, el Instituto Nacional de Salud Mental de Estados Unidos ha propuesto una estrategia de investigación cuyo punto de partida se sustenta en la identificación y el estudio de las dimensiones básicas de las disfunciones que se presentan de modo transversal en los trastornos mentales.

Palabras clave. Autismo. Categorización. Comorbilidad. DSM-5. Research Domain Criteria. TDAH. TEA. Trastorno mental.

\section{Introducción}

El 18 de mayo de 2013, tras una gran expectación y varios aplazamientos, salió a la luz el Manual diagnóstico y estadístico de los trastornos mentales, quinta edición (DSM-5). El proceso de elaboración se pudo seguir paso a paso desde la página web de la Asociación Americana de Psiquiatría (www. dsm5.org), que permitía a cualquier persona interesada seguir, día a día, los cambios que se iban incorporando al proyecto. El debate, muy activo, se centró, principalmente, en la clasificación y criterios diagnósticos para cada trastorno y en la nosología de las enfermedades mentales en general.

Los avances de la genética, de la biología celular, de la neuroimagen y de la neurofisiología, aun sin ofrecer respuestas definitivas, estaban cuestionando el concepto de trastorno mental y, a partir de aquí, aspectos nucleares del modelo conceptual del DSM. Steven E. Hyman, miembro del comité de revisión del DSM-5 y de la Clasificación Internacional de Enfermedades, décima revisión, comparó el conflicto generado durante la elaboración del DSM-5 al problema de un avión que debe ser reparado en pleno vuelo [1]. No sorprende, por tanto, que el DSM-5 saliera a la luz acompañado de un fuerte debate. Resulta innegable que el DSM, sobre todo a partir del DSM-III, no sólo ha llegado a ser un lenguaje común, aceptado por la mayor parte de la comunidad científica, sino que también ha facilitado notables avances terapéuticos. Pero, como contrapartida al gran desarrollo alcanzado, han ido emergiendo lagunas e incongruencias inherentes al modelo. La, por entonces, presidenta de la Asociación Americana de Psiquiatría manifestaba que el éxito alcanzado por el DSM había dado lugar a una grave consecuencia no deseada: los trastornos del DSM, después de cuatro décadas, habían llegado a ser considerados como entidades reales [2]. Es decir, afirmaba que los trastornos mentales, del modo como estaban concebidos, en realidad eran constructos artificiales carentes de identidad y que, por lo tanto, no podían ser homologables a las enfermedades que se podían objetivar mediante pruebas biológicas. Al carecer de una base sólida, el DSM había llegado a convertirse en un obstáculo para el progreso de investigación, afirmaba Donald Regier, subdirector del comité encargado de la elaboración del DSM-5 [3]. En este sentido era, y es, preocupante la desmotivación generada por la debilidad conceptual del modelo de cara a la búsqueda de nuevos recursos terapéuticos. A modo de ejemplo: GlaxoSmithKline, Novartis y AstraZeneca han abandonado la investigación para los fármacos psiquiátricos; Pfizer, Merck y Sanofi no la recomiendan [4]. En los últimos años (datos de 2011), sólo se están investigando 303 fármacos psiquiátricos frente a 3.436 tratamientos para el cáncer y 1.247 para otros trastornos neurológicos [5].

Ante este panorama, dos semanas antes de la publicación del DSM-5, Thomas R. Insel, director del
Centre Mèdic Psyncron; Sabadell, Barcelona (J. Artigas-Pallarés). Departamento de Métodos de Investigación y Diagnóstico en Educación, MIDE; Universitat de Barcelona; Barcelona, España (I. Paula-Pérez)

Correspondencia:

Dra. Isabel Paula Pérez. Campus Mundet. Departamento MIDE. Edif. Llevant, 2. piso, despacho 264. E-08035 Barcelona.

E-mail:

isabelpaula@ub.edu

Declaración de intereses: Los autores manifiestan la inexistencia de conflictos de interés en relación con este artículo.

Aceptado tras revisión externa: 15.01.15.

Cómo citar este artículo: Artigas-Pallarés J, Paula-Pérez I. Asignaturas pendientes del DSM-5. Rev Neurol 2015; 60 (Supl 1): S95-101.

(c) 2015 Revista de Neurología 
Instituto Nacional de Salud Mental (NIMH), entidad que financia en Estados Unidos los proyectos de investigación más importantes en salud mental, declaró que no serían financiados los estudios basados exclusivamente en los criterios del DSM. Y añadía que la investigación debía reorientarse al margen de las categorías del DSM, dado que su punto débil era la falta de validez de los diagnósticos, al estar basados en el consenso sobre agrupaciones sintomáticas carentes del soporte de pruebas biológicas objetivas [6].

Bruce N. Cuthbert, coordinador del proyecto Research Domain Criteria (RDoC) del NIMH, quizás motivado por el inmenso revuelo generado por la drástica y trascendente afirmación, salió al paso manifestando que el objetivo que perseguía el NIMH era estudiar los sistemas neuronales de forma directa, tomando en consideración su influencia transversal sobre diversos trastornos del DSM. También afirmaba que nos movemos hacia una nueva dirección, sin que ello quiera significar que a partir del próximo mes dejarían de ser aceptados los diagnósticos del DSM. Más bien se trataría de un cambio en el énfasis. O sea, en lugar de estudiar las entidades tal como están concebidas en el DSM, primaría la investigación sobre aspectos nucleares de la mente humana que intervienen en los trastornos mentales.

\section{Críticas al DSM-5}

Las críticas lanzadas por el NIMH, aun siendo las más radicales, no han sido las únicas ni tampoco las más divulgadas.

Un amplio grupo de psiquiatras, liderado por Alan Frances [7], director del DSM-IV, ha planteado fuertes objeciones, no tanto hacia el modelo general del DSM-5, como a aspectos puntuales relacionados con diversos trastornos. La aparición de nuevos diagnósticos, extremadamente comunes en la población general, podría haber estado promovida por intereses de la industria farmacéutica; tal es el caso del trastorno por bulimia, el déficit cognitivo leve y el trastorno por desregulación del estado de ánimo. Además, los criterios para ciertos trastornos, ya recogidos en el DSM-IV, se han suavizado de tal modo que se incrementa el número de pacientes diagnosticados. Por ejemplo, para el trastorno depresivo mayor, en el DSM-5, se exige que los síntomas estén presentes únicamente durante dos semanas en lugar de los seis meses del DSM-IV.

Otra fuente de críticas procede del colectivo de profesionales que, desde posiciones afines al movimiento de la antipsiquiatría, niegan la existencia de los trastornos psiquiátricos. De todos modos, esta crítica no es aplicable específicamente al DSM-5, sino a cualquier intento de codificar los problemas mentales.

Ante este panorama, John Hogan, cronista de la revista American Scientific, describía la situación actual manifestando que, a pesar de que el DSM haya sido considerado como la biblia de la salud mental, a lo sumo que puede aspirar es a ser un diccionario, la mera creación de un conjunto de etiquetas con una definición para cada una de ellas [8]. El impacto de sucesivas ediciones del DSM ha permitido que los médicos hayan llegado a utilizar los mismos términos con idéntico significado, con lo cual se ha alcanzado una buena fiabilidad, pero con una falta de validez. A diferencia de las definiciones de la enfermedad isquémica del corazón, del linfoma o del sida, basadas en su naturaleza, los diagnósticos del DSM se basan en un consenso acerca de los clusters de síntomas clínicos, pero sin ninguna medida objetiva. En el resto de la medicina, esto sería equivalente a la creación de sistemas de diagnóstico basados simplemente en la concurrencia de síntomas (imaginemos, por ejemplo, cuántas enfermedades cumplirían los criterios de fiebre, cefaleas, náuseas y vómitos). Por todo ello, el citado cronista afirmaba que los pacientes con trastornos mentales se merecían algo mejor.

\section{La falacia de la categorización}

El DSM-I [9] apareció en el año 1952, a raíz de las consecuencias de la Segunda Guerra Mundial, como un instrumento para desarrollar clasificaciones operativas necesarias para fijar las secuelas mentales en los veteranos de la guerra. La finalidad era dar respuesta a una exigencia administrativa en una época de gran confusión en el campo de la psiquiatría, cuando el debate se centraba en la causalidad psicológica o biológica de los trastornos mentales.

El psicoanálisis y el conductismo, pero especialmente el primero, tuvieron una fuerte influencia en las dos primeras versiones del DSM, donde se prefería utilizar el término reacción en lugar de trastorno. Los problemas mentales se consideraban 'reacciones' de la personalidad individual frente a factores sociales, biológicos y psicológicos. A partir del DSM-III [10], la balanza empezó a decantarse hacia la orientación biológica. La propuesta partía de la visión kraepeliniana de la enfermedad mental, donde se esperaba que, a partir de una identificación precisa de las entidades clínicas, se llegarían a 
esclarecer la etiología y la fisiopatología, del mismo modo que ocurre en la mayor parte de las enfermedades llamadas orgánicas. Sin embargo, en el DSM, a diferencia de los cuadros clínicos de las enfermedades conocidas, no se contempla ningún tipo de jerarquía entre los síntomas. El diagnóstico viene determinado por el número de criterios exigidos, al margen del peso específico que cada uno pueda aportar. Es decir, se definieron clusters de síntomas basándose únicamente en asociaciones estadísticamente significativas. El modelo de trastorno mental del DSM era, y sigue siendo, categórico. El umbral del número de criterios define la condición de tener o no el trastorno y, en consecuencia, la prueba que avala la inclusión en un grupo de estudio. Al margen de las distintas clasificaciones y criterios, los trastornos mentales se han incorporado al lenguaje popular y al científico como categorías; es decir, un individuo presenta, o no, determinado trastorno. Se es autista o no se es autista; se tiene trastorno por déficit de atención/hiperactividad (TDAH) o no se tiene TDAH; y así para el conjunto de entidades recogidas en el DSM.

Sin embargo, la simple observación de la fenomenología de los problemas mentales cuestiona la categorización. Un paciente puede entrar o salir fácilmente de un diagnóstico por varios motivos: la valoración subjetiva de cada síntoma - por parte del paciente y por parte del profesional-, la modificación de los criterios en distintas versiones del DSM, el uso del DSM o la Clasificación Internacional de Enfermedades, la elevada frecuencia con que se ubica un trastorno en la inestable franja límite del diagnóstico (codificada bajo el recurso acomodaticio de NOS). La categorización también cuestiona la existencia del llamado fenotipo ampliado que suelen presentar familiares de primer grado, lo cual, en realidad, no es otra cosa que la franja entre lo que se denomina trastorno y la normalidad. De todo ello se desprende que el modelo categórico resulta desconcertante para el paciente -dicotomía enfermo/no enfermo- y artificial para la investigación, al no encajar con la realidad.

\section{La fallacia de la comorbilidad}

De acuerdo con los patrones categóricos del DSM, un mismo individuo fácilmente puede cumplir, al mismo tiempo, los criterios para más de un trastorno. Se interpreta, entonces, que existe una comorbilidad; es decir, la presencia simultánea de dos o más trastornos. Por ejemplo, para el TDAH, el 6790\% de los pacientes presenta una comorbilidad y el
34-67\% al menos dos comorbilidades [11-13]. En general, la tasa de comorbilidad que proporciona el DSM es tal que un estudio puso en evidencia que los clínicos tienden a diagnosticar sólo una quinta parte de los diagnósticos que se obtendrían utilizando entrevistas semiestructuradas [14].

Para afirmar que dos o más trastornos son comórbidos se debería demostrar que ambos tienen una etiología distinta. Tomemos el ejemplo de la comorbilidad entre el TDAH y el trastorno del espectro autista (TEA). Resulta que el 22-83\% de niños con TEA comparte el diagnóstico de TDAH, mientras que el 30-65\% de pacientes con TDAH muestra síntomas de TEA [15]. Ante este alto grado de coincidencia, se hace difícil admitir que las bases genéticas, neuronales y cognitivas del TDAH y el TEA responden a mecanismos completamente distintos. Este ejemplo se puede aplicar a cualquier trastorno mental.

Resulta clarificador analizar un estudio llevado a cabo por el Psychiatric Genomics Consortium, en el cual se analiza una extensa base de datos acumulada a partir de diversos estudios de genome-wide associatión (GWA) en pacientes con esquizofrenia, trastorno bipolar, trastorno depresivo mayor, TDAH y autismo [16]. A causa del tamaño muestral relativamente bajo de cada uno de los citados estudios, los hallazgos fueron débiles o resultaron contradictorios respecto a resultados previos, por lo que se decidió acumular en un mismo grupo a todos los pacientes. El objetivo era el siguiente: si dichos trastornos, a pesar de corresponder a fenotipos distintos, compartieran mecanismos genéticos comunes, al acumularlos todos en un mismo grupo sería posible determinar significación estadística para variantes genéticas coincidentes. Se comparó una muestra de 33.332 casos con un grupo control de 27.888 individuos. Al disponer de un elevado número de casos, fue posible identificar con significación estadística en el grupo de pacientes los genes $C A C N A 1 C$ y $C A C N B 2$, ambos reguladores de los canales de calcio. El gen $C A C N A 1 C$ previamente ya se había hallado como un gen de susceptibilidad para el trastorno bipolar, la esquizofrenia y el trastorno depresivo mayor. Además, el gen CACNA1C está involucrado en el síndrome de Timothy, entre cuyas manifestaciones se incluye el autismo. Se sabe también que el CACNA1C está relacionado con la modulación de circuitos involucrados en el procesamiento de las emociones, funciones ejecutivas, atención y memoria. El CACNB2 interactúa con otras unidades $(C A C N A 1 C, C A C N A 1 D$ y $C A C N A 1 S)$ que intervienen en la permeabilidad selectiva de la membrana celular y modulan la función de otras proteínas y moléculas. La conclusión que se desprende de 
Tabla I. Sistemas de valencia negativa.

Amenaza aguda (miedo)

Amenaza potencial (ansiedad)

Amenaza sostenida

Pérdida

Frustración por falta de recompensa

este estudio es que la actividad de los canales de calcio se postula como un proceso biológico muy importante en relación con los trastornos psiquiátricos en general.

En fechas más recientes se ha podido llevar a cabo un estudio de GWA con una muestra de 36.989 casos de pacientes diagnosticados de esquizofrenia y 113.075 controles. Como era de esperar en una muestra de esta magnitud, se detectaron 108 loci, de los cuales el $75 \%$ incluye genes codificadores de proteínas. Entre los genes identificados se hallaban el DRD2 (diana de todos los medicamentos antipsicóticos eficaces), además de otros genes (por ejemplo, GRM3, GRIN2A, SRR, GRIA1) implicados en la neurotransmisión glutaminérgica y la plasticidad sináptica. En este estudio, al tratarse de una muestra grande de pacientes con esquizofrenia, se identificó, al igual que en el estudio citado anteriormente, la relación entre la esquizofrenia y los genes asociados a la actividad de los canales de calcio (CACNA1C, CACNB2 y CACNA1I). Como se ha señalado anteriormente, dichos genes están implicados, al igual que los genes reguladores de la neurotransmisión glutaminérgica y la plasticidad sináptica, tanto en la esquizofrenia como en otros trastornos psiquiátricos [17]. Igualmente enfatiza este artículo la coincidencia de variantes raras de gran tamaño (variaciones en el número de copias) halladas tanto en la esquizofrenia como en el autismo y la discapacidad intelectual [18]. A pesar del gran número de loci identificados en este estudio, los resultados distan mucho de aportar un patrón genético más o menos específico para la esquizofrenia, pues los autores estiman que tan sólo un 3,5\% de la varianza en la causalidad de la esquizofrenia se puede explicar por el efecto de los loci detectados mediante los estudios de GWA.

De todo ello se desprende que los mecanismos genéticos no son específicos para los trastornos mentales basados en la definición del DSM, ni tampoco lo son los mecanismos neurofuncionales a ni-
Tabla II. Sistemas de valencia positiva.

Motivación

Valoración de la recompensa

Valoración del esfuerzo/disponibilidad para trabajar

Error en la predicción de la recompensa

Selección de la atención/toma de decisiones basada en la preferencia

Capacidad de respuesta inicial a la recompensa

Capacidad de respuesta sostenida a la recompensa

Aprendizaje de la recompensa

Hábito

vel molecular. Adicionalmente, se puede inferir que los mecanismos cognitivos básicos, vinculados a la actividad de vías neuronales, difícilmente pueden configurar patrones neuropsicológicos específicos para determinado trastorno.

La investigación derivada del DSM se ha basado en el paradigma categórico y en un concepto artificial de comorbilidad. Pensemos, por un momento, que un grupo de estudio que cumple los criterios de TDAH es seleccionado para un estudio genético. Para conseguir unos resultados válidos se debería asumir que el grupo es homogéneo, o sea, que comparte una etiología genética común, por lo menos en una gran parte de los casos; y que, además, este grupo no está contaminado por otros trastornos comórbidos, con lo cual quedaría garantizada la pureza del grupo de estudio y podría ser comparado con una muestra de individuos no TDAH. Si se aceptan las elevadas cifras de comorbilidad obtenidas en diversos estudios, resulta que el TDAH 'puro' es un trastorno muy raro, donde resulta muy difícil y cuestionable que una muestra de TDAH no esté contaminada con otros trastornos. Además, tomemos ahora en consideración el carácter dimensional. Resultará que en el grupo control de pacientes no TDAH, un número muy importante presentará un TDAH subclínico (por ejemplo, un paciente con cinco criterios de inatención y cinco criterios de hiperactividad podría formar parte de un grupo control). Si a ello añadimos que el grupo control también tendrá sus 'comorbilidades', clínicas y subclínicas, resulta que la confusión es enorme y que los resultados obtenidos pueden resultar dudosos o erróneos. 
Tabla III. Sistemas cognitivos.

\begin{tabular}{l} 
Atención \\
\hline Percepción \\
Percepción visual \\
Percepción auditiva \\
Percepción olfativa somatosensorial multimodal \\
Memoria declarativa \\
Conducta lingüística
\end{tabular}

Control cognitivo (esfuerzo)

Selección del objetivo

Actualización

Representación y mantenimiento

Selección de la respuesta. Inhibición o supresión

Supervisión del rendimiento

\begin{tabular}{l} 
Memoria de trabajo \\
Mantenimiento activo \\
\hline Actualización flexible \\
Capacidad limitada \\
Control de la interferencia \\
\hline
\end{tabular}

\section{Propuesta del Instituto Nacional de Salud Mental: Research Domain Criteria}

La inconsistencia del modelo del DSM se puede sintetizar en la carencia de un marco nosológico con una sólida base científica que acredite la investigación. Si bien desde el punto clínico asistencial, el DSM -en tanto no se consolide una alternativa mejor- puede seguir siendo útil como lenguaje común para la práctica asistencial, en el campo de la investigación está obstaculizando el progreso [19, 20]. Si la investigación continúa sustentándose en categorías meramente descriptivas que no encajan con los mecanismos subyacentes [21], se seguirá fracasando tanto en la comprensión de la patogenia, como en la investigación de nuevos fármacos y en el desarrollo de pruebas diagnósticas. El motivo por el cual la investigación basada en el DSM ha alcanzado su límite se debe a que muchos factores de riesgo, genéticos, neuronales y ambientales [22] implicados en la fisiopatología de los trastornos men-
Tabla IV. Sistemas de procesos sociales.

$$
\begin{aligned}
& \text { Afiliación y apego } \\
& \text { Formación del apego y su mantenimiento } \\
& \text { Comunicación social } \\
& \text { Recepción de la comunicación facial } \\
& \text { Producción de comunicación facial } \\
& \text { Recepción de la comunicación no facial } \\
& \text { Producción de comunicación no facial }
\end{aligned}
$$

$$
\text { Percepción y comprensión de sí mismo }
$$

Identificación de los pensamientos y conductas de uno mismo

Conocimiento de sí mismo

$$
\begin{aligned}
& \text { Percepción y comprensión de los demás } \\
& \text { Percepción del comportamiento contingente } \\
& \text { Percepción de las acciones de los demás } \\
& \text { Comprensión de los estados mentales }
\end{aligned}
$$

Tabla V. Arousal y sistemas de regulación.

Arousal (activación emocional)

Ritmos circadianos

Sueño y vigilia

tales inciden de modo transversal en los diagnósticos del DSM.

La propuesta de los RDoC representa un cambio de paradigma que parte del conocimiento de la relación entre conductas observables y parámetros biológicos (neurofuncionales y genéticos). El modelo de los RDoC se plantea el objetivo de definir las dimensiones básicas de las disfunciones que intervienen de modo transversal en los trastornos mentales, contempladas tradicionalmente como categorías. Con ello se pretende desarrollar nuevas formas de clasificación, basadas en dominios de la conducta observable y en su relación con marcadores de posibles causas subyacentes y mecanismos implicados. Este enfoque asume que:

- El diagnóstico basado en la biología y los síntomas no debe estar constreñido por las categorías del DSM.

- Los trastornos mentales tienen un origen biológico que involucra circuitos cerebrales que se ex- 
presan mediante dominios específicos de la cognición, la emoción o la conducta.

- Utiliza varias unidades diferentes de análisis; por ejemplo, neuroimagen, actividad fisiológica y autoinformes de los síntomas.

- Se concibe como un sistema dimensional que parte de la normalidad.

- Cada nivel de análisis debe ser entendido como una dimensión de determinada función.

- El mapeo de las bases cognitivas, circuitos y aspectos genéticos de los trastornos mentales se espera que abra nuevos objetivos y facilite avances en el tratamiento [6].

Las dimensiones que configuran la matriz de los $\mathrm{RDoC}$ son: la función, las unidades de análisis, los aspectos vinculados al desarrollo y los aspectos ambientales. El desarrollo de la matriz debe ser un proceso dinámico, y las modificaciones en su estructura y contenido se irán generando a partir de la evidencia acumulada.

Los dominios en los que se basa la RDoC son:

- Sistemas de valencia negativa (Tabla I).

- Sistemas de valencia positiva (Tabla II).

- Sistemas cognitivos (Tabla III).

- Sistemas de procesamiento social (Tabla IV).

- Sistemas de activación emocional y regulatorios: activación, ritmo circadiano, vigilia y sueño (Tabla V).

\section{Conclusiones}

La predicción que se desprende de los datos recogidos en esta revisión de las críticas desencadenadas a partir de la elaboración del DSM-5 es la de la configuración de un nuevo paradigma que permita comprender los trastornos mentales en el marco de la nueva genética, alejada del modelo mendeliano; bajo una fisiopatología que desborda la concepción localizacionista de los trastornos mentales; y donde, además, no existen mecanismos cognitivos específicos. El final, si es que puede existir un final del camino que se abre, no parece estar a la vuelta de la esquina; por tanto, no sería sensato, en el ámbito de la práctica clínica, abandonar el modelo actual del DSM. El confusionismo que se generaría difícilmente se podría encauzar. Pero, al margen de los cambios que se avecinan en las estrategias de investigación, sí que parece importante que los profesionales implicados en la atención de pacientes cuyos problemas tienen actualmente cobertura en el DSM incorporemos en la práctica del día a día el pensamiento dimensional y cuestionemos, al menos co- mo concepto, la comorbilidad. Sin duda, ello facilitará al paciente una mejor comprensión de sus problemas y liberará al clínico de la servidumbre a una praxis diagnóstica y terapéutica excesivamente rígida y dogmática. Es preciso también matizar que la falta de validez de los diagnósticos del DSM-5 que se puede inferir a partir de los conocimientos actuales en modo alguno significa que algunos de los constructos diagnósticos actuales no puedan llegar a ser entidades operativas respaldadas por un conocimiento más preciso de las bases genéticas y neurofuncionales subyacentes.

Tampoco se debería interpretar que los RDoC tienen como objetivo sustituir un modelo de clasificación y definición de los problemas mentales, sino que, por el contrario intentan desbloquear el estancamiento que se deriva de una conceptualización no ajustada a la realidad. En este sentido, un cambio muy relevante, y que puede parecer insignificante, es el hecho de abandonar la numeración romana utilizada desde el DSM-I al DSM-IV-TR para adoptar la numeración arábiga: DSM-5. Esta modificación tiene como objetivo permitir que el DSM, en ediciones sucesivas, pueda incorporar de forma ágil los hallazgos que se vayan generando durante los próximos años, sin necesidad de tener que esperar una década, o más, hasta la aparición de una nueva versión global. El modelo de numeración actual va a permitir cambios parciales del contenido, incorporando los nuevos hallazgos: DSM-5.1, DSM-5.2, y así sucesivamente, hasta que se plantee un cambio radical que acumule los pequeños cambios.

Bibliografía

1. Hyman SE. Commentary: repairing a plane while it is flying -reflections on Rutter. J Child Psychol Psychiatry 2011; 52 : 661-2.

2. Bernstein CA. Meta-structure in DSM-5 process. Psychiatr News 2011; 46: 7.

3. AACAP. 59. ${ }^{a}$ Reunión Anual. 2012.

4. Abbott A. Novartis to shut brain research facility. Nature 2011; 480: 161-2.

5. Klein DF, Glick ID, Shader RI. Central nervous system drug development, basic, and clinical research: thinking outside the box. J Clin Psychopharmacol 2011; 31: 553-4.

6. Insel T. Transforming diagnosis. National Institute of Mental Health, 2013; URL: http://www.nimh.nih.gov/about/director/ 2013/transforming-diagnosis.shtml.

7. Frances A. Essentials of psychiatric diagnosis. New York: Guilford Press; 2013.

8. Horgan J. Psychiatry in crisis! Mental health director rejects psychiatric 'bible' and replaces with... nothing. URL: http:// blogs.scientificamerican.com/cross-check/2013/05/04/ psychiatry-in-crisis-mental-health-director-rejects-psychiatricbible-and-replaces-with-nothing/.

9. American Psychiatric Association. Diagnostic and statistical manual of mental disorders. Washington DC: APA; 1952.

10. American Psychiatric Association. Diagnostic and statistical manual of mental disorders, third edition. Washington DC: APA; 1980. 
11. Larson K, Russ SA, Kahn RS, Halfon N. Patterns of comorbidity, functioning, and service use for US children with ADHD, 2007. Pediatrics 2011; 127: 462-70.

12. Spencer T, Biederman J, Wilens T. Attention-deficit/hyperactivity disorder and comorbidity. Pediatr Clin North Am 1999; 46: 915-27.

13. Kadesjö B1, Gillberg C. The comorbidity of ADHD in the general population of Swedish school-age children. J Child Psychol Psychiatry 2001; 42: 487-92.

14. Basco M, Bostic JQ, Davies D, Rush AJ, Witte B, Hendrickse W, et al. Methods to improve diagnostic accuracy in a community mental health setting. Am J Psychiatry 2000; 157: 1599-605.

15. Artigas-Pallarés J. Autismo y TDAH: convergencias y divergencias. Genética. Rev Neurol 2013; 57 (Supl 1): S155-61.

16. Cross-Disorder Group of the Psychiatric Genomics Consortium. Identification of risk loci with shared effects on five major psychiatric disorders. Lancet 2013; 381: 1371-9.
17. Ferreira MA, O’Donovan MC, Meng YA, Jones IR, Ruderfer DM, Jones L, et al. Collaborative genome-wide association supports a role for ANK3 and CACNA1C in bipolar disorder. Nat Genet 2008; 40: 1056-8.

18. Sullivan PF, Daly MJ, O'Donovan M. Genetic architectures of psychiatric disorders: the emerging picture and its implications. Nat Rev Genet 2012; 13: 537-51.

19. Hyman SE. The diagnosis of mental disorders: the problem of reification. Annu Rev Clin Psychol 2010; 6: 155-179.

20. Craddock N, Owen MJ. The Kraepelinian dichotomy -going, going... but still not gone. Br J Psychiatry 2010; 196: 92-5.

21. Kapur S, Phillips AG, Insel TR. Why has it taken so long for biological psychiatry to develop clinical tests and what to do about it? Mol Psychiatry 2012; 17: 1174-9.

22. Owen MJ. Intellectual disability and major psychiatric disorders: a continuum of neurodevelopmental causality. Br J Psychiatry 2012; 200: 268-9.

\section{Unresolved issues in the DSM-5}

Summary. This paper analyses the criticism prompted by the publication of the Diagnostic and statistical manual of mental disorders, fifth edition (DSM-5), which was already foreseen to a certain extent during the final stages of its drafting. Part of the criticism has focused on the changes in the diagnostic criteria for certain disorders and the incorporation of new entities into the DSM. Another line of criticism, however, is aimed at the lack of diagnostic validity of the DSM, which has led to its efficiency in the research field being questioned. The basic underlying flaw in the DSM is the incoherence of a model based on a wide range of definitions of categorical entities, all of which have a high element of comorbidity. As a proposal to overcome the blockage generated in research and the scarcity of therapeutic advances, the US National Institute of Mental Health has put forward a research strategy based on identifying and studying the fundamental dimensions of the dysfunctions that present transversally in mental disorders.

Key words. ADHD. ASD. Autism. Categorisation. Comorbidity. DSM-5. Mental disorder. Research Domain Criteria. 